Title

\title{
Myocardial extracellular volume quantified by magnetic resonance is increased in cirrhosis and related to poor outcome
}

\section{Author names}

Signe Wiese ${ }^{1,2}$, Jens Hove ${ }^{3}$, Silje $\mathrm{Mo}^{2}$, Rajeshwar P. Mookerjee ${ }^{4}$, Claus L. Petersen ${ }^{1}$, Marianne K. VesterAndersen $^{5}$, Naja D. Mygind ${ }^{6}$, Jens P. Goetze ${ }^{7}$, Andreas Kjær ${ }^{1,8}$, Flemming Bendtsen ${ }^{2}$, Søren Møller ${ }^{1}$.

\section{Affiliations}

${ }^{1}$ Center of Functional Imaging and Research, Department of Clinical Physiology and Nuclear Medicine, Hvidovre Hospital, University of Copenhagen, Denmark.

${ }^{2}$ Department of Gastroenterology, Hvidovre Hospital, University of Copenhagen, Denmark.

${ }^{3}$ Department of Cardiology, Hvidovre Hospital, University of Copenhagen, Denmark.

${ }^{4}$ Liver Failure Group, UCL Institute for Liver and Digestive Health, UCL Medical School, Royal Free Hospital, United Kingdom

${ }^{5}$ Department of Hepatology, Rigshospitalet, University of Copenhagen, Denmark

${ }^{6}$ Department of Cardiology, Rigshospitalet, University of Copenhagen, Denmark

${ }^{7}$ Department of Clinical Biochemistry, Rigshospitalet, University of Copenhagen, Denmark

${ }^{8}$ Department of Clinical Physiology, Nuclear Medicine, PET and Cluster for Molecular Imaging,

Rigshospitalet, University of Copenhagen, Denmark

\section{Corresponding author:}

Signe Wiese, MD.

Center of Functional Imaging and Research, Department of Clinical Physiology and Nuclear Medicine,

Hvidovre Hospital, Kettegaard Alle 30, DK-2650 Hvidovre, Denmark.

E-mail: signe.wiese@ @ regionh.dk $\quad$ Telephone: +45 $38622461 \quad$ Fax: $\quad+4538623750$

\section{Electronic word count:}


Manuscript incl. title page, lay summary, abstract and references: 4996

\section{Number of figures and tables}

Figures: $2+1$ suppl.

Tables: 6

\section{List of abbre viations:}

CCM cirrhotic cardiomyopathy

ECV extracellular volume

CMR cardiac magnetic resonance imaging

$\mathrm{CO} \quad$ cardiac output

CI cardiac index

LVEF left ventricular ejection fraction

LAVI left atrial maximum volume index

DD diastolic dysfunction

Hs-TnT high sensitivity troponin $\mathrm{T}$

ProANP proatrial natriuretic peptide

ProBNP probrain natriuretic peptide

LT liver transplantation

HVPG hepatic venous pressure gradient

MELD model for end stage liver disease

\section{Conflict of interest: none}

\section{Financial support:}

This paper was supported by grants from the Novo Nordisk Foundation, Arvid Nilssons Foundation, and the Capital Region Research Foundation. 


\begin{abstract}
:
Background \& aims: The underlying pathogenesis of cirrhotic cardiomyopathy is unclear. Structural myocardial changes including diffuse fibrosis may be involved and can be accurately assessed by cardiac MRI (CMR) with quantification of the extracellular volume (ECV). This is the first application of this technique in patients with cirrhosis. We aimed to investigate the presence of diffuse myocardial fibrosis and to determine the relation to disease severity, cardiac function, and outcome.
\end{abstract}

Methods: A prospective study including 52 cirrhotic patients and 10 healthy controls. All patients underwent CMR with ECV quantification, tissue Doppler echocardiography, and biochemical assessments. Patients were follow-up for a median of 25 months with registration of death and liver transplantation (LT).

Results: Myocardial ECV was higher in the patients compared with healthy controls (31.2 \pm 6 vs. $27.4 \pm 3 \%, P=0.04)$. Furthermore, ECV increased across the Child Pugh $\mathrm{A} / \mathrm{B} / \mathrm{C}$ classes $(26.9 \pm 4 /$ $31.5 \pm 5 / 34.4 \pm 6 \%, P=0.02$ ). Four-teen patients experienced the composite end-point of death/LT during follow-up and these patients had higher $\mathrm{ECV}(33.2 \pm 4$ vs. $30.4 \pm 6 \%, P=0.04)$. In a univariate Cox regression analysis ECV was associated with poor transplant-free survival (HR 3.6 [1.1-11.6]; $P=0.03$ ). However, MELD and CRP remained the strongest predictors in a multivariate analysis. ECV correlated with cardiac index $(\mathrm{r}=0.44, P=0.001), \mathrm{CRP}(\mathrm{r}=0.46, P=0.001)$, proANP $(\mathrm{r}=0.50$, $P<0.001)$, and proBNP (r=0.40, $P=0.005)$.

Conclusions: Myocardial ECV is increased in patients with cirrhosis and seems related to disease severity and transplant-free survival. These changes most likely reflect subclinical diffuse myocardial fibrosis and may represent a structural element of cirrhotic cardiomyopathy. Key words: Liver disease; Cirrhotic cardiomyopathy; Myocardial fibrosis; T1 mapping 
Electronic word count of the abstract: 244

\section{Lay summary:}

A magnetic resonance scan of the heart reveals changes in the cardiac wall of patients with end stage liver disease. These structural changes seem to worsen in relation to disease severity and impact on prognosis.

\section{Introduction}

Cardiac dysfunction in cirrhosis is receiving increasing attention. The term Cirrhotic cardiomyopathy (CCM) describes a clinical entity of impaired systolic responsiveness to stress, diastolic dysfunction, and electrophysiological abnormalities observed in patients with cirrhosis ${ }^{1}$. Importantly, CCM has been associated with the development of renal failure and poor prognosis. However, the underlying pathophysiology of CCM is still largely unresolved. Results from autopsy studies suggest that structural cardiac abnormalities including diffuse myocardial fibrosis may be involved $^{2}$. Cardiac magnetic resonance $(\mathrm{CMR})$ with measurement of the longitudinal relaxation (T1) times is able to assess myocardial tissue characteristics such as infiltration, edema, and fibrosis. The quantification of the myocardial extracellular volume fraction (ECV) by use of native and post-contrast $\mathrm{T} 1$ maps has been histologically validated with a strong correlation to diffuse myocardial fibrosis ${ }^{3}$. Myocardial ECV has been shown to be a strong predictor of poor outcome in several cardiac diseases including cardiomyopathies, heart failure, and diabetes ${ }^{4}$. The present study is the first to apply myocardial ECV quantification in patients with cirrhosis. The purpose of this study was to use CMR with myocardial ECV quantification to explore the presence of diffuse myocardial fibrosis in patients with cirrhosis. We hypothesized that CMR-derived myocardial ECV 
is increased in cirrhosis indicating diffuse myocardial fibrosis, and that myocardial ECV relates to disease progression, cardiac function, and poor outcome.

\section{Patients and Methods}

Study design. A prospective, observational study in a cohort of patients with cirrhosis performed at Hvidovre Hospital, Denmark. Written informed consent was obtained from all patients before study enrollment. The study protocol conformed to the ethical guidelines of the 1975 Declaration of Helsinki and was approved by the Scientific Ethics Committee of the Capital Region of Denmark (H-4-2013-045).

Patient population. A total of 115 patients with cirrhosis attending the Hepatology outpatient clinics at Hvidovre Hospital and Rigshospitalet in the period from September 2014 to February 2017 were evaluated for enrollment. Inclusion criteria were: Age 18-75 years and a diagnosis of cirrhosis either based on histology or clinical, biochemical and ultrasonography findings. Patients were excluded if they had pre-existing cardiac or respiratory disease $(n=9)$, type 1 diabetes $(n=3)$, ongoing infections $(n=3)$, impaired renal function (estimated glomerular filtration rate $<45$ $\left.\mathrm{ml} / \mathrm{min} / 1,73 \mathrm{~m}^{2}\right)(\mathrm{n}=6)$, previous transjugular intrahepatic portosystemic shunt or liver transplantation $(n=2)$, malignancy $(n=2)$, anti-viral hepatitis $C$ medication $(n=5)$, contraindications to CMR or contrast-agents $(n=5)$, refused participation $(n=7)$ or were considered incapable to adhere to the study protocol due to heavy drinking $(n=10)$. All patients underwent clinical evaluation, blood sample collection, 12-lead ECG, tissue Doppler echocardiography, contrastenhanced CMR and a CT coronary angiography in the same day. The latter was obtained in order to exclude presence of undiscovered ischemic heart disease. In order to reduce confounding factors diuretics and beta-blockers were withdrawn $48 \mathrm{~h}$ prior to the investigations. The cohort included a total of 63 patients; however ECV quantification was not performed in 11 patients due to poor 
compliance in the scanner, or lack of intravenous access. These patients were excluded for this substudy. A group of 10 healthy age and sex matched subjects without known cardiovascular risk factors and with a completely normal CMR scan, served as controls. The control group did not undergo further investigations.

\section{Cardiac magnetic resonance imaging}

Cine CMR. All participants received clinical CMR scans by dedicated CMR technologists with a 3.0-Tesla Siemens Magnetom Verio (Siemens Medical Solutions, Erlangen, Germany) and a 32channel phased array cardiovascular coil. The examination included standard breath hold segmented cine imaging with steady state free precession. Left and right ventricular dimensions, myocardial mass, cardiac output $(\mathrm{CO})$ and cardiac index $(\mathrm{CI})$ were measured from short axis stacks of end diastolic and end systolic cine frames.

T1 mapping and ECV quantification. T1 mapping was performed with electrocardiographically triggered MOLLI with a 5(3)3 prototype on a short-axis view. This method generates an inline, pixel-based T1 map by acquiring a series of images over several heartbeats with shifted T1 times, inline motion correction, and inline calculation of the $\mathrm{T} 1$ relaxation curve within one breath hold. T1-sequence parameters were as follows: starting inversion time (TI) $120 \mathrm{~ms}$, TI increment $80 \mathrm{~ms}$, reconstructed matrix size $360 \times 307$ ( phase-encoding resolution 66\%, phase-encoding field of view 85.2\%). T1 maps with motion correction were created before and 8 min after gadolinium application. For post-contrast T1 mapping, a 4(1)3(1)2 prototype was used. Post processing was performed using dedicated software (cmr42, Circle Cardiovascular Imaging Inc., Calgary, Canada). $\mathrm{T} 1$ values (in milliseconds) were obtained by drawing regions of interest around the entire myocardium in a single midventricular slice and in the blood pool on the corresponding pre- and post-contrast images. Care was taken to secure a sufficient distance to tissue interfaces. ECV was 
calculated with the formula ${ }^{5}: \mathrm{ECV}$ in $\%=\chi \mathrm{x}(1-$ hematocrit $)$. Where $\chi=(\Delta \mathrm{R} 1$ myocardium $) / \Delta \mathrm{R} 1$ blood $)$ and $\Delta \mathrm{R} 1=(1 /$ post-contrast $\mathrm{T} 1)-(1 /$ pre-contrast $\mathrm{T} 1)$. Normal values were obtained in the 10 healthy volunteers.

Reproducibility of myocardial ECV. For intraobserver variability, the same operator repeated the measurements in 25 studies in anonymized form, more than 8 weeks after the initial analysis. For interobserver variability, a second operator re-analyzed the images of the entire cohort in anonymized form. Intra- and interobserver variability of ECV was $0.1 \pm 2.4 \%$ and $0.4 \pm 2.9 \%$, respectively.

Echocardiography. Transthoracic tissue Doppler echocardiography was performed by two experienced echocardiographers using a commercially available system (Epiq 7G, Phillips, Andover, USA). Left ventricular inflow velocities were determined using pulsed-wave Doppler with a $4 \mathrm{~mm}$ sample placed between the tips of the mitral leaflets during diastole at end-expiration. The following parameters were measured: isovolumetric relaxation time, peak early filling and its deceleration time, atrial filling peak, and E/A ratio. Mitral annulus tissue velocities (e') were acquired by pulse-wave tissue-Doppler imaging with the sample volume placed at the septal and lateral mitral annulus. The E/e' ratio was calculated using the average e'. The left atrial volume was determined by area-length method from end-systolic apical four-chamber and two-chamber views. Left atrial maximum volume index (LAVI) was averaged from both views and corrected for body surface area. Left ventricular diastolic dysfunction (DD) was then classified into the following 4 categories: normal diastolic function, E/A ratio $>1$ and $\mathrm{E} / \mathrm{e}^{\prime}$ ratio $<8$; grade 1 , E/A ratio $<0.75$ and $\mathrm{E} / \mathrm{e}^{\prime}$ ratio $<8$; grade 2, E/A ratio $0.75-1.5$ and $\mathrm{E} / \mathrm{e}^{\prime}$ ratio 8 -15; and grade 3, $\mathrm{E} / \mathrm{A}$ ratio $>1.5$ and $\mathrm{E} / \mathrm{e}^{\prime}$ ratio > 15. Analysis was performed off-line with dedicated software (Q-lab vs 10.1.1., Phillips, Andover, MA, USA). 
Cirrhotic cardiomyopathy criteria. Patients were diagnosed with CCM if they fulfilled the criteria for diastolic dysfunction and/or had a left ventricular ejection fraction (LVEF) $\leq 55 \%$ indicative of systolic dysfunction.

Biomarkers. Blood samples were obtained from the cubital vein, and plasma was stored at $-80{ }^{\circ} \mathrm{C}$. High-sensitivity troponin $\mathrm{T}$ (hs- $\mathrm{TnT}$ ) and probrain natriuretic peptide (proBNP) were measured on an automated Modular E platform (Roche, Mannheim, Germany). Mid-regional proatrial natriuretic peptide (proANP) was measured on a Kryptor Plus platform (Thermo-Fisher, Waltham, USA). Copeptin (provasopressin) was analyzed with a standard sandwich immuno-luminometric assay (Thermo-Fisher, Waltham, USA Brahms). We have previously reported on assay performance and characteristics $^{6}$. Renin was estimated with a commercially available immunoradiometric assay (DGR International, Hamburg, Germany). LLQ was $0.31 \mathrm{~g} / \mathrm{mL}$, intra- and inter-assay imprecision was $2 \%$. Noradrenaline and adrenaline were measured as the stable metabolites methoxynoradrenaline and methoxy-adrenaline using a commercially available kit (Acquity UPLC IClass Xevo TQ-S; Waters Corporation, USA) with a LLQ of $0.1 \mathrm{nmol} / \mathrm{L}$.

Follow-up registration and poor outcome. All patients were prospectively followed up at 6-month intervals by ambulatory visits, telephone calls or review of the medical files. Poor outcome was defined as the composite end point of liver transplantation (LT) and death.

Statistics. Data were stored and analyzed using SPSS Statistics 22 (IBM Corp., NY, USA). The results are reported as median $\left(25^{\text {th }}-75^{\text {th }}\right.$ percentile), mean $\pm \mathrm{SD}$ or as $\mathrm{n}(\%)$ as appropriate. The Student $t$, Mann-Whitney, or chi-squared tests were used to compare continuous or categorical variables. One-way analysis of variance (ANOVA) was used to compare multiple groups. In cases of non-normality, the non-parametric Kruskal-Wallis ANOVA was applied. Correlations were performed by the Spearman rank test to investigate possible interrelation between variables. 
Follow-up data was analyzed by using Kaplan Meier plots with log-rank tests for group comparisons. Univariate and multivariate proportional hazard Cox regression analyses were performed to evaluate the association of myocardial ECV and well-known prognostic variables with transplant-free survival. All reported $P$ values are two-tailed with values less than 0.05 considered statistically significant. Patients were divided into two groups according to their myocardial ECV to separate those with the highest ECV, and compared with respect to cardiac function, poor outcome, and transplant-free survival. The cut off for purposes of dividing the patients was pragmatically chosen as the mean myocardial ECV $31.2 \%$, though supported by the range of abnormal ECV values reported in patients with cardiomyopathies, systemic inflammation and diabetes ${ }^{4,7}$.

\section{Results}

The study included 52 patients with cirrhosis. In Table 1 is listed the clinical and biochemical characteristics of the patients. The age (median $63,(52 ; 67))$ and sex $(6$ male, $50 \%)$ of healthy controls were not significantly different from those of the patients. Most patients belonged to Child Pugh class B $(n=37,71 \%)$. The etiology of cirrhosis was alcohol consumption in the majority of the patients (77\%). Two patients had a combined etiology of hepatitis $\mathrm{C}$ and alcohol. Eighteen patients had abstained from alcohol > 6 months. Portal hypertension had been established by liver vein catheterization prior to enrollment in 39 patients with a median hepatic venous gradient (HVPG) of $16 \mathrm{mmHg}(12 ; 20)$. Forty-one patients were treated with diuretics, 22 with beta-blockers, and two patients also received statins. No patients were treated with other cardiovascular drugs (e.g. ACE-inhibitors, angiotensin II receptor blockers or acetylsalicylic acid). CMR-derived structural and functional parameters are listed in Table 2. Patients with cirrhosis had a higher cardiac index than healthy controls, whereas no significant differences were seen in respect to the 
cardiac dimensions. Patients with advanced cirrhosis (Child Pugh > 7) displayed markedly increases in end-diastolic volumes of both left and right ventricle resulting in an increased stroke volume, $\mathrm{CO}$ and $\mathrm{CI}$ in comparison to patients with less advanced cirrhosis (Child Pugh $\leq 7$ ). Myocardial ECV was increased in patients compared with healthy controls $(31.2 \pm 6$ vs. $27.4 \pm 3 \%, P=0.04)$. Moreover, myocardial ECV was higher in patients with advanced cirrhosis in comparison with both healthy controls and patients with less advanced cirrhosis as shown in Figure 1. Additionally, myocardial ECV increased across Child Pugh classes (26.9 $\pm 4 / 31.5 \pm 5 / 34.4 \pm 6 \%, P=0.02)$. No significant differences in myocardial ECV were seen neither when data was stratified according to alcohol vs. non-alcohol etiology, use of beta-blockers or statins, nor according to established cardiovascular risk factors such as smoking, diabetes, arterial hypertension, and hypercholesterolemia $(P=\mathrm{NS})$. Echocardiographic parameters are listed in Table 3. Left ventricular DD was present in 19 patients $(37 \%)$ with grade 1 DD in one patient and grade 2 DD in the remaining. No patients had grade 3 DD. Moreover, left atrial enlargement (LAVI $>34 \mathrm{ml} / \mathrm{m}^{2}$ ) was present in 21 patients $(33 \%)$ and an increased E/e' ratio $\geq 10$ in 17 patients (32\%) pointing towards an increased left ventricular filling pressure. Resting systolic function was impaired (LVEF<55\%) in 2 patients, one of these also had DD. Accordingly; CCM was diagnosed in 20 patients (38\%).

ECV in relation to markers of disease severity and cardiac dysfunction. Bivariate correlations between myocardial ECV and clinical, biochemical, echocardiographic, and CMR-derived parameters are shown in Table 4. Myocardial ECV correlated with markers of disease severity including model for end stage liver disease (MELD) ( $\mathrm{r}=0.31, P=0.03)$, albumin $(\mathrm{r}=-0.40, P=0.004)$, and hemoglobin $(\mathrm{r}=-0.55, P<0.001)$. ECV correlated with CRP ( $\mathrm{r}=0.46, P=0.001)$, and when patients were stratified in two groups according to CRP > $10 \mathrm{mg} / \mathrm{l} \mathrm{ECV}$ was markedly higher in those with an increased CRP $(34.3 \pm 5$ vs. $30.3 \pm 5 \%, P=0.03)$. Myocardial ECV correlated with cardiac function: Cardiac index $(\mathrm{r}=0.44, P=0.001)$, lateral e' $(\mathrm{r}=0.43, P=0.002), \mathrm{E}(\mathrm{r}=0.37$, 
$P=0.006)$, and E/A ratio $(\mathrm{r}=0.28, P=0.05)$. No relation between ECV and presence of DD. Patients with an increased E/e' ratio tended to have a higher myocardial ECV, however this difference was not statistically significant $(33.2 \pm 6$ vs. $30.5 \pm 5 \%, P=\mathrm{NS})$. The following cardiovascular markers correlated with myocardial ECV: ProANP ( $\mathrm{r}=0.50, P<0.001)$, proBNP $(\mathrm{r}=0.40, P=0.005)$, and renin $(\mathrm{r}=0.31, P=0.03)$. Initially there was no significant correlation between ECV and hs-TnT. However, when patients were stratified according to a median hs-TnT $>20 \mathrm{ng} / \mathrm{L}$ those with a higher level of circulating hs-TnT had a markedly increased ECV (37.2 \pm 4 vs. $30.6 \pm 5 \%, P=0.01)$.

ECV and poor outcome. During a median follow-up of 25 months (total range:1-34 months)14 patients $(27 \%)$ experienced the composite end-point of death $(n=10)$ or LT $(n=4)$. Patients with a poor outcome had a higher myocardial ECV than those without $(33.2 \pm 4$ vs. $30.4 \pm 6 \%, P=0.04)$. The LT patients had a higher mean myocardial ECV than those who died, although not statistically significant ( $34.8 \pm 8$ vs. $31.7 \pm 3 \%, P=\mathrm{NS}$ ). Table 5 compares patients with a high myocardial ECV (>31.2\%) to those with a low ECV $(\leq 31.2 \%)$. Patients with a high ECV differed with respect to the following: Lower albumin, higher CRP, higher levels of natriuretic peptides, a higher cardiac output and cardiac index, increased E/A ratio, E and lateral e' $(P<0.05)$. The use of beta-blockers or statins did not affect neither the outcome nor the presence of increased myocardial ECV, $P=\mathrm{NS}$. Transplant-free survival was significantly greater in patients with a low myocardial ECV compared to those with a high myocardial ECV ( 86 vs. $57 \%, p=0.02$ ). Figure 2 shows the probability of transplant-free survival stratified according to myocardial ECV with a lower transplant-free survival in the high ECV group. Moreover, if patients were stratified both according to their median MELD and myocardial ECV, those with a high MELD and a high myocardial ECV tended to have a poorer transplant-free survival (Suppl. Figure S1). In Table 6 is shown univariate proportional hazard Cox regression analyses and a high myocardial ECV was associated with increased risk of poor outcome $\mathrm{HR}=3.6(95 \% \mathrm{CI}=1.1-11.6), P=0.03$. However, when $\mathrm{ECV}$ and well-known prognostic variables 
in cirrhosis were included in a multivariate proportional hazard Cox regression analysis MELD and CRP remained the strongest markers independently associated with poor outcome. The causes of death were: Sepsis $(n=4)$, hepatorenal failure $(n=1)$, liver failure/cirrhosis $(n=1)$, alcoholic hepatitis $(n=1)$, intracerebral bleeding $(n=1)$, and unknown in 2 patients who were found dead at home. Interestingly, myocardial ECV was high in the patients with unknown cause of death $(33 \%$ and $35 \%$, respectively). These patients also had increased levels of natriuretic peptides, and QTc was prolonged in one $(468 \mathrm{~ms})$ and borderline in the other $(438 \mathrm{~ms})$.

\section{Discussion}

This study is the first to investigate expansion of the myocardial extracellular volume in patients with cirrhosis using CMR. We show that patients with cirrhosis have an increased myocardial ECV. We also establish a significant relationship between myocardial ECV and severity of the liver disease. Finally, an increased myocardial ECV seemed to be related to poor prognosis. These findings may therefore provide new insight into the pathophysiology of CCM since they substantiate an underlying substrate for structural myocardial abnormalities in the absence of clinical symptoms. Additionally, our data demonstrate relations between myocardial ECV and proANP, proBNP, and hs-TnT, which are all markers of cardiac strain and dysfunction. These associations further add to the assumption that an increased myocardial ECV indicates myocardial abnormalities in patients with cirrhosis and may therefore represent a structural element of CCM. Structural cardiac changes such as swelling of the myocytes, hypertrophy, altered collagen composition, and interstitial fibrosis have all been suggested to contribute to the underlying pathophysiology of CCM based on findings in autopsy and experimental studies ${ }^{2,8}$. Few clinical studies have investigated structural cardiac changes in cirrhosis. Lossnitzer et al. performed CMR with late gadolinium enhancement in 20 patients with end stage liver disease and described a pattern of focal fibrosis resembling the appearance of myocarditis ${ }^{9}$. Sampaio et al. recently 
performed a dobutamine stress CMR including late gadolinium enhancement imaging in 36 patients with mild to moderate cirrhosis, but did not find significant focal myocardial fibrosis. Instead they reported inotropic incompetence to pharmacological stress with abnormal myocardial strain indicating intrinsic myocardial abnormalities ${ }^{10}$. No previous studies have performed T1-mapping and myocardial ECV quantification in patients with cirrhosis. However, the technique was recently applied in patients with viral hepatitis $\mathrm{C}$ and moderate liver disease with the finding of abnormal $\mathrm{T} 1$ values indicating diffuse myocardial fibrosis ${ }^{11}$.

ECV and inflammation. It is well-established that a chronic systemic low-grade inflammation is present in cirrhotic patients. We found a significant association between myocardial ECV and CRP with significantly higher ECV in patients with an increased CRP. Our results could indicate that low-grade inflammation in cirrhosis is associated with development of structural myocardial changes. Recent studies have supported a similar relationship of increased myocardial ECV in patients with rheumatoid arthritis, diabetes, and sarcoidosis which are all conditions associated with inflammation ${ }^{4}$. Moreover, inflammation is known to play an important role in sepsis-induced cardiomyopathy and one could speculate that comparable pathophysiological processes are involved in the development of CCM. In fact, experimental studies have coupled an inflammatory milieu with increased TNF- $\alpha$ levels and cytokine-induced stimulation of nitric oxide production to cardiac dysfunction in cirrhosis, but the exact mechanisms have not been elucidated ${ }^{12}$. The finding of a relatively low CRP in our cohort may be explained by the selection of stable cirrhotic patients, and the exclusion of patients with ongoing bacterial infections. Therefore, in future studies it would be interesting to assess myocardial ECV in patients with a pronounced inflammatory activity.

ECV and systolic dysfunction. The hyperdynamic circulation has been proposed as an important element for the development of CCM. The high cardiac output is a result of the vasodilatory state and tends to maintain a sufficient perfusion in the vital organs. However, this may inflict cardiac 
strain due to the increased cardiac work which over time causes the development of cardiac dysfunction $^{1}$. In our study the relationship between markers of a hyperdynamic circulation and myocardial ECV was ambiguous, but we revealed a strong association between myocardial ECV on the one hand and a high cardiac index and increased plasma renin levels on the other. This may indicate important pathophysiological implications, which need to be investigated further. A resting systolic dysfunction was only present in two patients and both of these patients had a normal myocardial ECV. A plausible explanation to this finding is that our study population consisting primarily of stable cirrhotic outpatients in whom systolic dysfunction was only latent. In future studies it would be relevant to apply pharmacological stress in order to unmask subclinical systolic impairment.

ECV and diastolic dysfunction. Post-contrast T1-times have been related to DD with a close correlation to E/e' in patients with hypertrophic cardiomyopathy ${ }^{13}$. Similarly, a recent study in cardiac transplant patients reported an association between an increased myocardial ECV and increased left ventricular wall stiffness, thereby linking intrinsic myocardial alterations to the mechanism of $\mathrm{DD}^{14}$. We found no relations between myocardial ECV and DD in our study, although the myocardial ECV of patients with an increased E/e' tended to be higher. It is however most likely that our stable cirrhotic patients from a hemodynamic point of view are different from those of patients with hypertrophic cardiomyopathy or cardiac transplant, respectively. It may therefore be a key issue to study the relationship between myocardial ECV and DD in patients with more advanced cirrhosis as recent studies have reported pronounced DD in these patients ${ }^{15,16}$.

ECV and poor outcome. An unfavorable relationship exists between myocardial ECV and outcome in patients with non-ischemic cardiomyopathy as well as in diabetes in terms of development of heart failure, cardiac mortality, and overall survival ${ }^{4}$. Similarly, we found indications pointing towards an association between increased ECV and poor outcome in patients with cirrhosis. We 
defined poor outcome as the combined endpoint of death or LT as we consider the need of organ replacement as an indicator of markedly disease deterioration. However, in the multivariate Cox regression analysis MELD and CRP remained the strongest independent predictors of survival, which is not surprising as both disease severity and inflammation repeatedly have been shown to have great impact on survival in cirrhosis ${ }^{17}$. It is important to be aware that the statistical power may not be sufficiently strong to perform a final evaluation of the relationship between myocardial ECV and outcome measures because of the limited number of endpoints. Therefore, the seemingly associated poor outcome needs to be confirmed in larger studies. The absence of an association between ECV and portal hypertension may in part be due to the time gap between measurements of HVPG and cardiac measures, which in some patients were more than 6 months.

Limitations. Although our study is a prospective study with application of advanced cardiac imaging techniques it has some limitations. Firstly, this is a single center study in a selected study population comprising predominantly stable outpatients due to several exclusion criteria. These criteria resulted in a considerable number of screening failures, but were chosen in order to eliminate potential confounders on cardiac function and survival. Moreover, the majority of our patients had cirrhosis on the basis of excessive alcohol intake. Thus our results may not be applicable to the general population of cirrhotic patients and need to be validated in larger studies including patients with more advanced cirrhosis and non-alcoholic etiologies. Secondly, we used a 3.0 T MR scanner which is more prone to artefacts due to the strong magnetic field. However, the mean ECV value in our healthy controls was similar to the $3.0 \mathrm{~T}$ reference values reported by others $^{18}$. This indicates that our ECV estimates are reliable. Third, we cannot verify that the increased myocardial ECV in cirrhotic patients is solely due to presence of diffuse fibrosis as presence of edema and infiltration may also expand the myocardial extracellular space thereby leading to an increased ECV. However, CMR-derived ECV correlates well to histologically diffuse 
myocardial fibrosis in endomyocardial biopsies from patients with non-ischemic heart failure ${ }^{19}$. Since these patients and cirrhotic patients share the clinical feature of fluid accumulation we believe that these results are transferable to cirrhosis. Furthermore, our myocardial ECV values are similar to those reported in both patients with cardiomyopathies (ECV: 31-41\%), systemic inflammation (ECV: 30\%), and with diabetes (ECV: $30.2 \%)^{4,7}$. Therefore, we consider the differences in myocardial ECV reported in our study of clinical significance, but confirmation in future studies is needed.

\section{Conclusion}

Myocardial ECV as determined by CMR is increased in cirrhotic patients indicating an expansion of the myocardial extracellular volume. These changes most likely reflect subclinical diffuse myocardial fibrosis and seem associated with disease severity. In addition the structural changes seem to possibly influence the prognosis. Hence, these preliminary findings indicate myocardial abnormalities as an element of CCM and the potential implication of this finding should be further explored.

Acknowledgment: We are indebted to our experienced echocardiographer Allan Poulsen, cardiologist Hanne Halgreen, bioanalyst Katrine Lyngby, and their colleagues at the Department of Clinical Physiology and Nuclear Medicine, for their invaluable assistance. Similarly, we acknowledge the huge effort made by the MRI technicians at Center for Diagnostic Imaging and Research.

This paper was supported by grants from the Novo Nordisk Foundation, Arvid Nilssons Foundation, and from the Capital Region Research Foundation. 


\section{References:}

1. Moller S, Henriksen JH, Møller S, Henriksen JH. Cirrhotic cardiomyopathy. J Hepatol. 2010;53:179-190.

2. Ortiz-Olvera NX, Castellanos-Pallares G, Gomez-Jimenez LM, et al. Anatomical cardiac alterations in liver cirrhosis: an autopsy study. AnnHepatol.2011;10:321-326.

3. Moon JC, Messroghli DR, Kellman P, et al. Myocardial T1 mapping and extracellular volume quantification: a Society for Cardiovascular Magnetic Resonance (SCMR) and CMR Working Group of the European Society of Cardiology consensus statement. J Cardiovasc Magn Reson. 2013;15:92.

4. Puntmann VO, Peker E, Chandrashekhar Y, NagelE. T1 Mapping in Characterizing Myocardial Disease: A Comprehensive Review. Circ Res. 2016;119:277-299.

5. Kellman P, Wilson JR, Xue H, Ugander M, Arai AE. Extracellular volume fraction mapping in the myocardium, part 1: evaluation of an automated method. J Cardiovasc Magn Reson. 2012;14:63.

6. Wiese S, Mortensen C, Gøtze JPJP, et al. Cardiac and proinflammatory markers predict prognosis in cirrhosis. Liver Int. 2014;34:e19-30.

7. Wong TC, Piehler KM, Kang IA, et al. Myocardial extracellular volume fraction quantified by cardiovascular magnetic resonance is increased in diabetes and associated with mortality and incident heart failure admission. Eur Heart J. 2014;35:657-664.

8. Glenn TK, Honar H, Liu H, ter Keurs HEDJ, Lee SS. Role of cardiac myofilament proteins titin and collagen in the pathogenesis of diastolic dysfunction in cirrhotic rats. J Hepatol. 2011;55:1249-1255.

9. Lossnitzer D, Steen H, Zahn A, et al. Myocardial late gadolinium enhancement cardiovascular magnetic resonance in patients with cirrhosis. J Cardiovasc.Magn Reson. 2010;12:47-.

10. Sampaio F, Lamata P, Bettencourt N, et al. Assessment of cardiovascular physiology using dobutamine 
stress cardiovascular magnetic resonance reveals impaired contractile reserve in patients with cirrhotic cardiomyopathy. J Cardiovasc Magn Reson. 2015;17:61.

11. Ngu PJ, Butler M, Pham A, Roberts SK, Taylor AJ. Cardiac remodelling identified by cardiovascular magnetic resonance in patients with hepatitis $\mathrm{C}$ infection and liver disease. Int J Cardiovasc Imaging. 2016;32:629-636.

12. Liu H, Lee SS. Nuclear factor-kappaB inhibition improves myocardial contractility in rats with cirrhotic cardiomyopathy. Liver Int. 2008;28:640-8.

13. Ellims AH, Iles LM, Ling L, Hare JL, Kaye DM, Taylor AJ. Diffuse myocardial fibrosis in hypertrophic cardiomyopathy can be identified by cardiovascular magnetic resonance, and is associated with left ventricular diastolic dysfunction. J Cardiovasc Magn Reson. 2012;14:76.

14. Ellims AH, Shaw JA, Stub D, et al. Diffuse myocardial fibrosis evaluated by post-contrast T1 mapping correlates with left ventricular stiffness. J Am Coll Cardiol. 2014;63:1112-1118.

15. Raevens S, De Pauw M, Geerts A, et al. Prevalence and outcome of diastolic dysfunction in liver transplantation recipients. Acta Cardiol. 2014;69:273-80.

16. Cesari M, Frigo AC, Tonon M, Angeli P. Cardiovascular predictors of death in patients with cirrhosis. Hepatology. 2017. doi:10.1002/hep. 29520

17. Gustot T, Fernandez J, Garcia E, et al. Clinical Course of acute-on-chronic liver failure syndrome and effects on prognosis. Hepatology. 2015;62:243-252.

18. Dabir D, Child N, Kalra A, et al. Reference values for healthy human myocardium using a T1 mapping methodology: results from the International T1 Multicenter cardiovascular magnetic resonance study. $J$ Cardiovasc Magn Reson. 2014;16:69.

19. aus dem Siepen F, Buss SJ, Messroghli D, et al. T1 mapping in dilated cardiomyopathy with cardiac magnetic resonance: quantification of diffuse myocardial fibrosis and comparison with endomyocardial 
biopsy. Eur Heart J Cardiovasc Imaging. 2015;16:210-216.

Figure Legends:

Figure 1: Myocardial extracellular volume fraction (ECV) quantified by cardiac magnetic resonance in patients with cirrhosis and healthy controls. Myocardial ECV is increased in patients with advanced cirrhosis (Child Pugh >7) both in comparison to healthy controls and patients with less advanced cirrhosis (Child Pugh $\leq 7$ ).

Figure 2: Kaplan Meier plot of the transplant-free survival in patients with cirrhosis stratified according to myocardial extracellular volume fraction (ECV). Patients with cirrhosis and a high myocardial ECV (> 31.2\%) have a poorer transplant-free survival than those with a low myocardial ECV $(\leq 31.2 \%)$.

Supplementary Figure S1: Kaplan Meier plot of the transplant-free survival in patients with cirrhosis stratified according to both MELD and myocardial extracellular volume fraction (ECV). Cirrhotic patients with a higher than median MELD (>10) and a high myocardial ECV (> $31.2 \%)$ tend to have a poorer transplant- free survival. 
Table 1: Clinical and biochemical characteristics of 52 patients with cirrhosis grouped according to Child Pugh score

\begin{tabular}{|c|c|c|c|c|}
\hline & $\begin{array}{l}\text { All patients } \\
\qquad(\mathrm{n}=52)\end{array}$ & $\begin{array}{c}\text { CP score }>7 \\
(n=21)\end{array}$ & $\begin{array}{c}\text { CP score } \leq 7 \\
(n=31)\end{array}$ & $P$ \\
\hline Age & $57(50 ; 64)$ & $(51 ; 64)$ & $57(50 ; 65)$ & 0.63 \\
\hline Male sex & $34(65)$ & $14 \quad(67)$ & $20(65)$ & 0.87 \\
\hline Etiology (alcohol/nonalcohol/combined) & $40 / 10 / 2$ & $15 / 6 / 0$ & $25 / 4 / 2$ & 0.21 \\
\hline MELD & $10(8 ; 13)$ & $(10 ; 16)$ & $8(7 ; 10)$ & 0.000 \\
\hline Mean arterial pressure $(\mathrm{mmHg})$ & $93(87 ; 100)$ & $(87 ; 96)$ & $97(85 ; 110)$ & 0.13 \\
\hline Heart rate $(\mathrm{bpm})$ & $73(65 ; 83)$ & $(66 ; 81)$ & $76(65 ; 85)$ & 0.53 \\
\hline $\mathrm{HVPG}^{\$}(\mathrm{mmHg})$ & $16(12 ; 20)$ & $(14 ; 20)$ & $15(10 ; 19)$ & 0.21 \\
\hline Beta-blocker therapy & $22(42)$ & (33) & $15(48)$ & 0.28 \\
\hline \multicolumn{5}{|l|}{ Biochemical analysis } \\
\hline Hemoglobin $(\mathrm{mmol} / \mathrm{L})$ & $7.9(6.7 ; 8.4)$ & $6.7(6.4 ; 8.2)$ & $8.1(7.4 ; 8.7)$ & 0.003 \\
\hline Creatinine $(\mu \mathrm{mol} / \mathrm{L})$ & $68(59 ; 76)$ & $65(61 ; 79)$ & $68(57 ; 76)$ & 0.67 \\
\hline Sodium (mmol/L) & $138(135 ; 141)$ & $137(134 ; 139)$ & $140(137 ; 141)$ & 0.02 \\
\hline INR & $1.3(1.1 ; 1.4)$ & $1.4(1.3 ; 1.6)$ & $1.2(1.1 ; 1.3)$ & 0.000 \\
\hline Total bilirubin $(\mu \mathrm{mol} / \mathrm{L})$ & $18(10 ; 30)$ & $41(18 ; 54)$ & $15(9 ; 19)$ & 0.001 \\
\hline Albumin $(g / L)$ & $31 \quad(26 ; 35)$ & $26(24 ; 29)$ & $33(30 ; 37)$ & 0.000 \\
\hline $\mathrm{CRP}(\mathrm{mg} / \mathrm{L})$ & $4(2 ; 10)$ & $6(4 ; 15)$ & $3(2 ; 5)$ & 0.005 \\
\hline \multicolumn{5}{|l|}{ Cardiovascular markers } \\
\hline proBNP (pmol/L) & $10(5 ; 24)$ & $19(7 ; 31)$ & $10(5 ; 15)$ & 0.05 \\
\hline proANP (pmol/L) & $92(70 ; 142)$ & $115(81 ; 189)$ & $84(66 ; 116)$ & 0.05 \\
\hline $\mathrm{Hs}-\mathrm{TnT}$ (ng/L) & $13(13 ; 14)$ & $13(13 ; 15)$ & $13(13 ; 13)$ & 0.14 \\
\hline Copeptin (pmol/L) & $7(4 ; 12)$ & $8(6 ; 13)$ & $7(4 ; 10)$ & 0.31 \\
\hline Renin (ng/L) & $23(8 ; 78)$ & $40(11 ; 131)$ & $18(7 ; 58)$ & 0.05 \\
\hline Methoxy-adrenaline ( $\mu \mathrm{mol} / \mathrm{L})$ & $0.18(0.12 ; 0.24)$ & $0.19(0.13 ; 0.24)$ & $0.17(0.11 ; 0.24)$ & 0.56 \\
\hline Methoxy-noradernaline $(\mu \mathrm{mol} / \mathrm{L})$ & $0.47(0.36 ; 0.65)$ & $0.44(0.30 ; 0.70)$ & $0.48(0.39 ; 0.59)$ & 0.84 \\
\hline QTc (ms) & $421(412 ; 439)$ & $422(413 ; 445)$ & $419(410 ; 431)$ & 0.28 \\
\hline
\end{tabular}

Results are presented as median $\left(25^{\text {tn }}\right.$ and $75^{\text {tn }}$ percentile) or number of patients (\%). 
${ }^{*}$ Autoimmune, viral, PSC, PBC, NASH, alfa-1-antrypsin deficiency, and cryptogenic. ${ }^{\$}$ HVPG data available in 39 patients.

CP: Child Pugh score; Hs-TnT: high sensitivity troponin T; HVPG: hepatic venous pressure gradient; MELD: model for end stage liver disease; proANP: proatrial natriuretic peptide; proBNP: probrain natriuretic peptide 
Table 2: Cardiac magnetic resonance parameters including myocardial extracellular volume (ECV) in patients with cirrhosis grouped according to Child Pugh score and in healthy controls

\begin{tabular}{|c|c|c|c|c|c|c|}
\hline & \multirow{2}{*}{$\begin{array}{l}\text { Patients } \\
(n=52)\end{array}$} & \multirow{2}{*}{$\begin{array}{l}\text { Controls } \\
(n=10)\end{array}$} & \multirow{2}{*}{$\begin{array}{l}\mathrm{CP}>7 \\
(\mathrm{n}=21)\end{array}$} & \multirow{2}{*}{$\begin{array}{l}\mathrm{CP} \leq 7 \\
(\mathrm{n}=31)\end{array}$} & \multicolumn{2}{|c|}{$P$} \\
\hline & & & & & $\begin{array}{c}\text { Patients vs. } \\
\text { controls }\end{array}$ & $\begin{array}{l}\text { Between } \\
\text { CP groups }\end{array}$ \\
\hline LV end-diastolic volume (ml) & $135 \pm 43$ & $137 \pm 39$ & $154 \pm 49$ & $122 \pm 34$ & 0.90 & 0.007 \\
\hline LV end-systolic volume (ml) & $43 \pm 17$ & $48 \pm 21$ & $48 \pm 20$ & $39 \pm 13$ & 0.37 & 0.05 \\
\hline RV end-diastolic volume (ml) & $134 \pm 45$ & $134 \pm 45$ & $152 \pm 48$ & $121 \pm 38$ & 0.98 & 0.02 \\
\hline RV end-systolic volume (ml) & $49 \pm 20$ & $46 \pm 21$ & $54 \pm 22$ & $45 \pm 17$ & 0.75 & 0.13 \\
\hline Stroke volume (ml) & $92 \pm 31$ & $89 \pm 20$ & $106 \pm 34$ & $83 \pm 25$ & 0.74 & 0.007 \\
\hline Cardiac output (L/min) & $6.8 \pm 2.4$ & $5.4 \pm 1.1$ & $7.6 \pm 2.5$ & $6.2 \pm 2.1$ & 0.07 & 0.04 \\
\hline Cardiac index $\left(\mathrm{L} / \mathrm{min} / \mathrm{m}^{2}\right)$ & $3.5 \pm 1.1$ & $2.8 \pm 0.5$ & $3.9 \pm 1.2$ & $3.3 \pm 1.0$ & 0.05 & 0.04 \\
\hline Myocardial mass index $\left(\mathrm{g} / \mathrm{m}^{2}\right)$ & $58 \pm 13$ & $55 \pm 8$ & $60 \pm 15$ & $56 \pm 12$ & 0.58 & 0.21 \\
\hline Myocardial ECV (\%) & $31.2 \pm 5.5$ & $27.4 \pm 2.9$ & $33.6 \pm 5.7$ & $29.6 \pm 4.8$ & 0.04 & 0.008 \\
\hline
\end{tabular}

Results are presented as mean \pm SD.

CP: Child Pugh score; LV: left ventricle; RV: right ventricle 
Table 3: Echocardiographic parameters in patients with cirrhosis grouped according to Child Pugh score

Patients $(n=52) \quad$ CP $>7(n=21) \quad$ CP $\leq 7(n=31)$

\begin{tabular}{lcccc}
\hline LV ejection fraction (\%) & $68 \pm 8$ & $69 \pm 8$ & $68 \pm 8$ & 0.62 \\
E/A ratio & $1.3 \pm 0.4$ & $1.3 \pm 0.4$ & $1.2 \pm 0.4$ & 0.19 \\
Deceleration time (ms) & $230 \pm 50$ & $231 \pm 47$ & $229 \pm 53$ & 0.81 \\
Isovolumetric relaxation time (ms) & $79 \pm 13$ & $79 \pm 12$ & $79 \pm 14$ & 0.99 \\
E $(\mathrm{cm} / \mathrm{s})$ & $75 \pm 19$ & $76 \pm 17$ & $74 \pm 20$ & 0.62 \\
Lateral e' $(\mathrm{cm} / \mathrm{s})$ & $11.6 \pm 2.8$ & $12.6 \pm 2.5$ & $10.8 \pm 2.7$ & 0.01 \\
Septal e' $(\mathrm{cm} / \mathrm{s})$ & $8.6 \pm 2.2$ & $9.2 \pm 2.1$ & $8.2 \pm 2.3$ & 0.08 \\
E/e' ratio & $8.3 \pm 2.8$ & $7.7 \pm 2.6$ & $8.7 \pm 2.9$ & 0.13 \\
LA volume index $\left(\mathrm{ml} / \mathrm{m}^{2}\right)$ & $32.1 \pm 8.0$ & $35.0 \pm 8.2$ & $30.2 \pm 7.3$ & 0.03 \\
\hline
\end{tabular}

Results are presented as mean \pm SD.

CP: Child Pugh score; LA: left atrial; LV: left ventricle 
Table 4: Spearman correlations between myocardial extracellular volume and clinical, biochemical, and cardiovascular parameters in patients with cirrhosis

\begin{tabular}{|c|c|c|}
\hline & Rho & $P$ \\
\hline Age & -0.11 & 0.46 \\
\hline MELD & 0.31 & 0.03 \\
\hline HVPG & 0.22 & 0.17 \\
\hline MAP & -0.21 & 0.14 \\
\hline Cardiac index & 0.44 & 0.001 \\
\hline Hemoglobin & -0.55 & 0.000 \\
\hline Albumin & -0.40 & 0.004 \\
\hline CRP & 0.46 & 0.001 \\
\hline $\mathrm{E} / \mathrm{A}$ ratio & 0.28 & 0.05 \\
\hline$E$ & 0.37 & 0.006 \\
\hline IVRT & -0.25 & 0.07 \\
\hline E/e' ratio & 0.06 & 0.68 \\
\hline Lateral e' & 0.43 & 0.002 \\
\hline LA volume index & 0.09 & 0.54 \\
\hline ProANP & 0.50 & 0.000 \\
\hline ProBNP & 0.40 & 0.005 \\
\hline Renin & 0.31 & 0.03 \\
\hline QTC & 0.09 & 0.55 \\
\hline
\end{tabular}

HVPG: hepatic venous pressure gradient; IVRT: isovolumetric relaxation time; LA: left atrial; MAP:

mean arterial pressure; MELD: model for end stage liver disease; proANP: proatrial natriuretic peptide; proBNP: probrain natriuretic peptide 
Table 5: Baseline measurements and outcome in patients with cirrhosis with a high myocardial extracellular volume (ECV) versus patient with cirrhosis with a low myocardial ECV

\begin{tabular}{|c|c|c|c|}
\hline Variables & High ECV $(n=23)$ & Low ECV $(n=29)$ & $P$ \\
\hline Age & $57(52 ; 64)$ & $56(50 ; 65)$ & 0.86 \\
\hline Alcohol etiology $(n, \%)$ & $18(78)$ & $22(82)$ & 0.77 \\
\hline Child Pugh score & $8(7 ; 10)$ & $7(7 ; 7.5)$ & 0.003 \\
\hline MELD & $10(8 ; 14)$ & $9(8 ; 11)$ & 0.09 \\
\hline HVPG (mmHg) & $17(11 ; 20)$ & $15(12 ; 19)$ & 0.47 \\
\hline Beta-blocker therapy $(\mathrm{n}, \%)$ & $7(30)$ & $15(52)$ & 0.12 \\
\hline Albumin (g/L) & $28(26 ; 33)$ & $33(29 ; 37)$ & 0.03 \\
\hline CRP (mg/L) & $6(4 ; 14)$ & $3(2 ; 5)$ & 0.002 \\
\hline \multicolumn{4}{|l|}{ Cardiovascular risk factors } \\
\hline Hypercholesterolemia (n, \%) & $2(9)$ & $7(24)$ & 0.14 \\
\hline Diabetes type II $(n, \%)$ & $4(17)$ & $6(21)$ & 0.76 \\
\hline Smoking $(n, \%)$ & $15(65)$ & $18(62)$ & 0.82 \\
\hline \multicolumn{4}{|l|}{ Cardiac MRI data } \\
\hline LV end-diastolic volume (ml) & $149(109 ; 164)$ & $119(96 ; 150)$ & 0.04 \\
\hline LV end-systolic volume (ml) & $43(34 ; 56)$ & $38(30 ; 48)$ & 0.16 \\
\hline Cardiac output (L/min) & $7.1(6.1 ; 9.0)$ & $5.8(4.8 ; 6.8)$ & 0.008 \\
\hline Cardiac index $\left(\mathrm{L} / \mathrm{min} / \mathrm{m}^{\mathrm{L}}\right)$ & $3.8(3.2 ; 4.6)$ & $3.0(2.5 ; 3.4)$ & 0.001 \\
\hline Myocardial mass index $\left(\mathrm{g} / \mathrm{m}^{2}\right)$ & $59(52 ; 66)$ & $55(46 ; 61)$ & 0.18 \\
\hline \multicolumn{4}{|l|}{ Echocardiographic data } \\
\hline LV ejection fraction (\%) & $68(62 ; 75)$ & $67(63 ; 73)$ & 0.73 \\
\hline LA volume index $\left(\mathrm{ml} / \mathrm{m}^{2}\right)$ & $31.2(26 ; 39)$ & $30.2(25 ; 38)$ & 0.65 \\
\hline E/A ratio & $1.2(1.1 ; 1.6)$ & $1.1(0.95 ; 1.3)$ & 0.04 \\
\hline Lateral e' (cm/s) & $12.6(10.6 ; 14.7)$ & $10.6(8.8 ; 11.8)$ & 0.001 \\
\hline \multicolumn{4}{|l|}{ Cardiovascular markers } \\
\hline ProBNP (pmol/L) & $15(8 ; 29)$ & $7(5 ; 13)$ & 0.03 \\
\hline ProANP (pmol/L) & $133(82 ; 189)$ & $81(63 ; 99)$ & 0.002 \\
\hline Hs-TnT (ng/L) & $13(13 ; 16)$ & $13(13 ; 13.4)$ & 0.26 \\
\hline Copeptin (pmol/L) & $8(5 ; 13)$ & $7(4 ; 10)$ & 0.17 \\
\hline
\end{tabular}


Adverse outcome (n, \%)

Results are presented as median $\left(25^{\mathrm{tI}}\right.$ and $75^{\mathrm{th}}$ percentile) or number of patients. High myocardial ECV was defined as ECV $>31.2 \%$ and low myocardial ECV as ECV $\leq 31.2 \%$

Hs-TnT: high sensitivity troponin T; HVPG: hepatic venous pressure gradient; LA: left atrial; LT: liver transplantation; LV: left ventricle; MELD: model for end stage liver disease; proANP: proatrial natriuretic peptide; proBNP: probrain natriuretic peptide 
Table 6: Parameters significantly associated with transplant-free survival at uni- and multivariate proportional hazard Cox regression analyses

\begin{tabular}{llll}
\hline Variables & $\mathrm{HR}$ & $95 \% \mathrm{Cl}$ & $P$ \\
\hline Univariate analysis & & & \\
MELD & 1.30 & $1.10-1.55$ & $\mathbf{0 . 0 0 3}$ \\
Child Pugh score & 1.61 & $1.20-2.16$ & $\mathbf{0 . 0 0 2}$ \\
CRP & 1.06 & $1.03-1.10$ & $\mathbf{0 . 0 0 0}$ \\
Myocardial ECV & 3.61 & $1.12-11.62$ & $\mathbf{0 . 0 3}$ \\
Multivariate analysis & & & \\
MELD & 1.25 & $1.04-1.52$ & $\mathbf{0 . 0 2}$ \\
CRP & 1.06 & $1.02-1.10$ & $\mathbf{0 . 0 0 5}$ \\
\hline ECV: extracellular volume; & MELD: modified end-stage liver disease
\end{tabular}




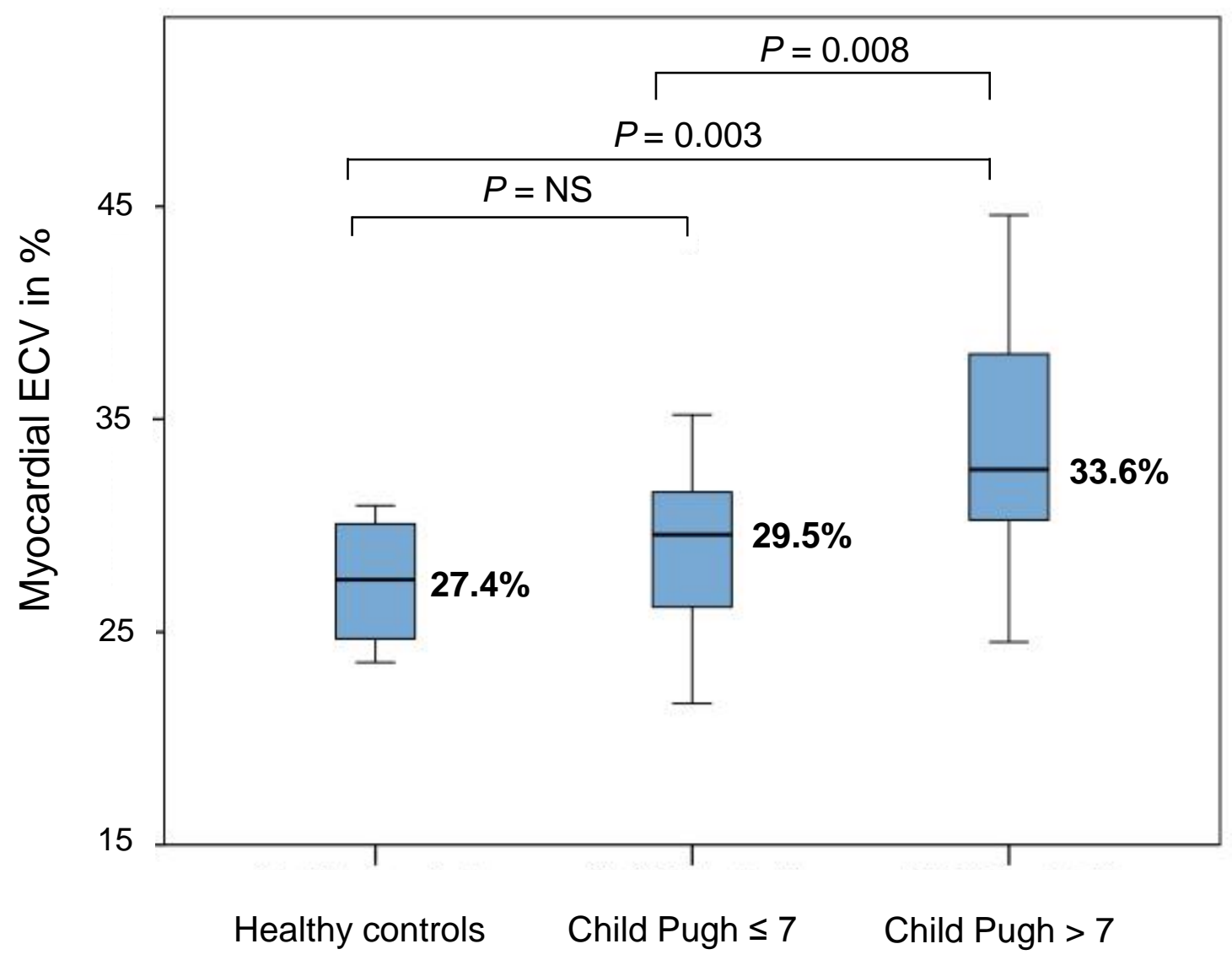




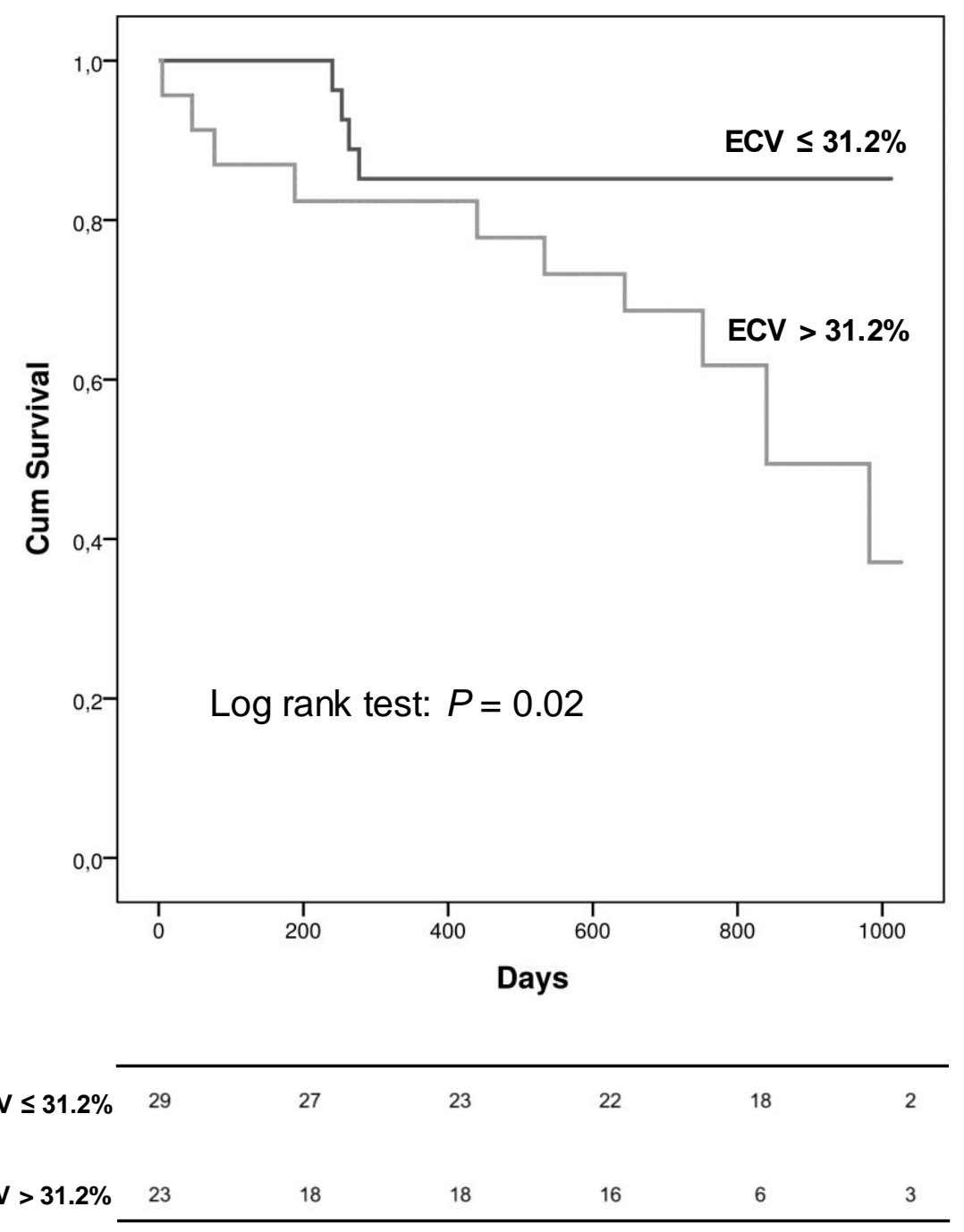




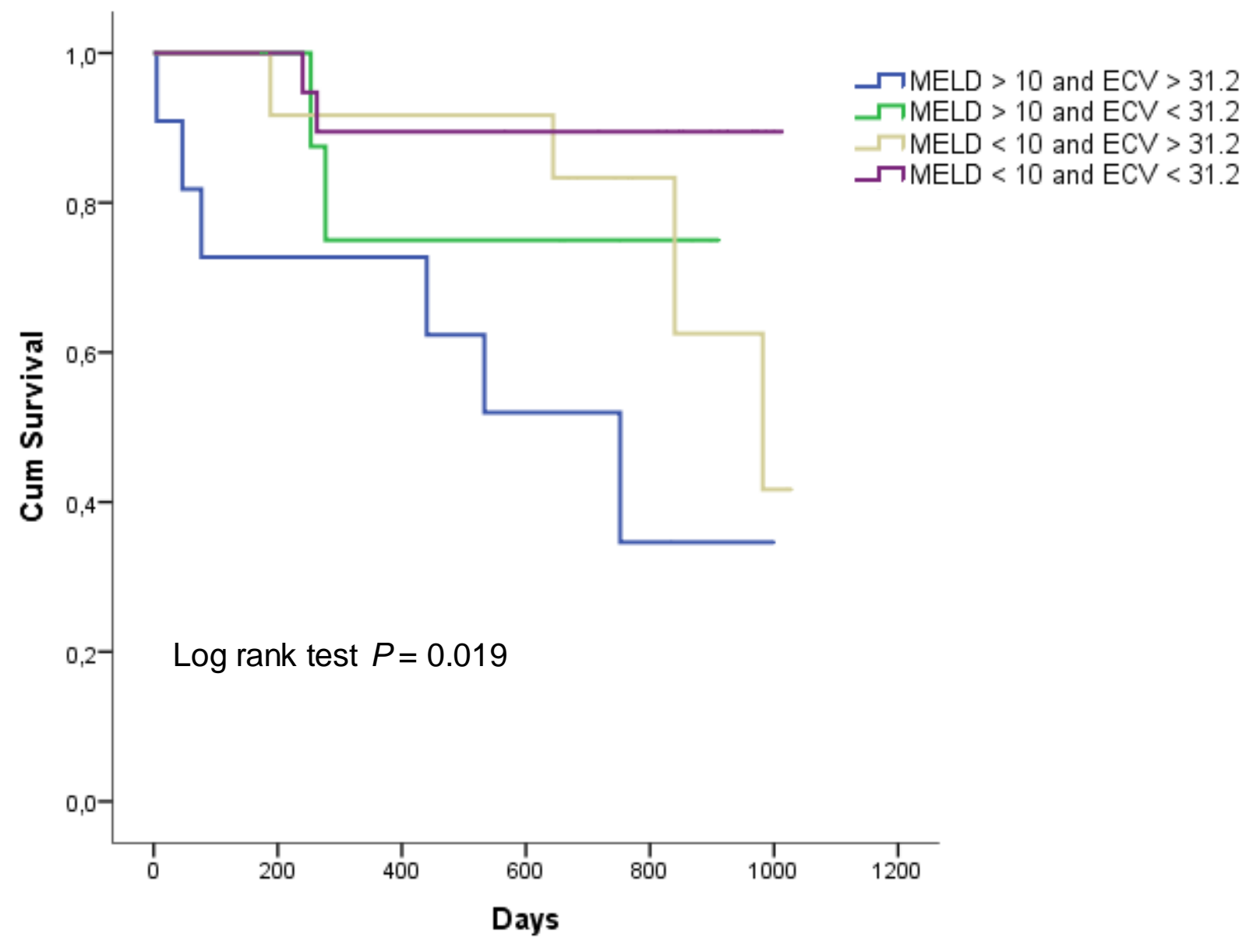

